\title{
A Multi Dimensional Visualization and Analysis Toolkit for Astrophysics
}

\author{
Daniela Ferro ${ }^{1}$, Vincenzo Antonuccio-Delogu ${ }^{1}$, Ugo Becciani ${ }^{1}$, Angela \\ Germaná $^{1}$, Claudio Gheller ${ }^{2}$, and Maura Melotti ${ }^{2}$ \\ 1 INAF Astrophisical Observatory of Catania, via S.Sofia 78, Catania 95100, Italy, \\ dferro@ct.astro.it, \\ http://woac.ct.astro.it/ \\ 2 CINECA, via Magnanelli 6-3, \\ Casalecchio di Reno BOLOGNA, Italy
}

\begin{abstract}
The AstroMD toolkit is a visualization and analysis software, specifically oriented to astrophysical data representation. It is included in the project Cosmo.Lab, financed by the European Community, which involves several European astrophysical institutions and CINECA. This tool gives a 3D graphic representation of the data exploiting all the available information and making use of the immersive visualization techniques. AstroMD uses the most advanced visualization technology, based on virtual reality, in order to build a leading edge instrument both for scientific research and for cultural dissemination and education. It is developed using the Visualization Toolkit (VTK) by Kitware (http://www.kitware.com), a freely available visualization library portable on several platforms. AstroMD is an open source completely free code which is freely available (http://cosmolab.cineca.it).
\end{abstract}

\section{Introduction}

Since the beginning of modern astronomy, the scientific community expressed a great interest in scientific visualization tools. A great improvement in this direction was determined by the introduction of modern CCD detectors to collect observational data in a digital format. Today, almost all the standard measures are digital and each measure can be generally considered as a collection of images forming a multi-dimensional data set. In many cases extensive image processing is required to obtain meaningful images. Useful scientific information can be obtained from the raw data only using a data reduction pipeline. The improvement of technology and the availability of super-computing multiprocessor systems, has led to a dramatic increase of the volume of data coming from numerical simulations. Today, astrophysical simulations produce many gigabytes of data which have to be efficiently visualized and analysed. Visualization is the most intuitive approach to the data and basic information can be obtained just "at a glance". Then the possibility of moving inside the data allows the scientist to focus on regions of interest and there to perform quantitative calculations. Therefore image processing tools are of fundamental importance in astronomy. 
AstroMD is a new data visualization and analysis software specifically projected to deal with astrophysical data and can handle powerfully large datasets allowing both their graphical representation and analysis, responding to the requirements proposed by several research fields. The basic application on which the new software is applied focus on the dataset of the VIRMOS (observational galaxy catalogue) project, the data coming from observations of extragalactic radio sources and those obtained by cosmological simulations. Although these fields do not cover all the requirements of astronomy, they pose many typical problems that we expect to be solved by AstroMD and therefore represent a significant test-case. The solution to these problems was implemented following the suggestions and the indications of the research groups involved in the project and of a User Interest group. Astrophysical data have peculiarities that make them different from data coming from any other kind of simulation or experiment. Therefore they require a specific treatment. For example, cosmological simulations consider both baryonic matter (described by fluid-dynamics) and dark matter (described by N-body algorithms). Further components, like stars or different chemical species, can be introduced and followed in a specific way. These different species requires different types of visualization. Dark matter needs particles position or velocity rendering while baryons require mesh based visualization. Furthermore particle associated quantities, like the mass density or the gravitational potential, require their calculation and visualization on a mesh. Then simulated structures have a fully three-dimensional distribution. Therefore it is necessary to have a clear 3D representation and efficient and fast tools of navigation, selection, zoom and the possibility of improving the resolution and the accuracy of calculations in specific, user-selected, regions. Moreover evolution can change dramatically the properties of the simulated objects and the information that can be retrieved, therefore it is important to control efficiently sequences of time-frames.

The scientific goals of the project are the study of the evolution of field galaxies, the evolution of large scale structure, the evolution of galaxies in clusters and the search for distant quasars. For extragalactic radio sources AstroMD provides tools to guide the understanding of the emissivity, flow and field structure, a process which is currently limited by our ability to compute rapidly and visualize the results. In cosmological simulations, one tries to reproduce the formation, evolution and the properties of large scale structures of the universe.

\section{The Software}

In order to build a widely used product it was chosen to use a low cost software portable on a number of different platforms, the Visualization Toolkit (VTK) by Kitware [4]. VTK is an open source, freely available software system for 3D computer graphics, image processing, and visualization. It includes a $\mathrm{C}++$ class library and several interpreted interface layers. VTK has been ported on nearly every Unix-based platform (e.g. Linux or IRIX) and PC's (Windows NT and Windows 98). The design and implementation of the library has been strongly 
influenced by object-oriented principles. The graphical model in VTK is at a higher level of abstraction than rendering libraries like OpenGL or PEX. This means it is much easier to create useful graphics and visualization applications. In VTK applications can be written directly in $\mathrm{C}++$, Tcl, Java, or Python. Using these languages it is possible to build powerful, fast and portable applications. The built-in functionalities are controlled by a specific Graphic User Interface (Fig 2), written in incrTcl/Tk that contains the OO philosophy, slowly adding code as new functionality are added to the package. Each object can represent a reader, that allows to read data from a file or from a database, a filter, that allows to manipulate data, or a viewer, that allows to visualize the results. VTK supports a wide variety of visualization algorithms including scalar, vector, tensor, texture, and volumetric methods and advanced modelling techniques. It supports stereographic rendering and can be used for virtual reality visualization [?]. Furthermore, being easily extensible, the system allows ad hoc implementation of specific modules. All the features described above are integrated in the AstroMD package. Furthermore efficient manipulation and

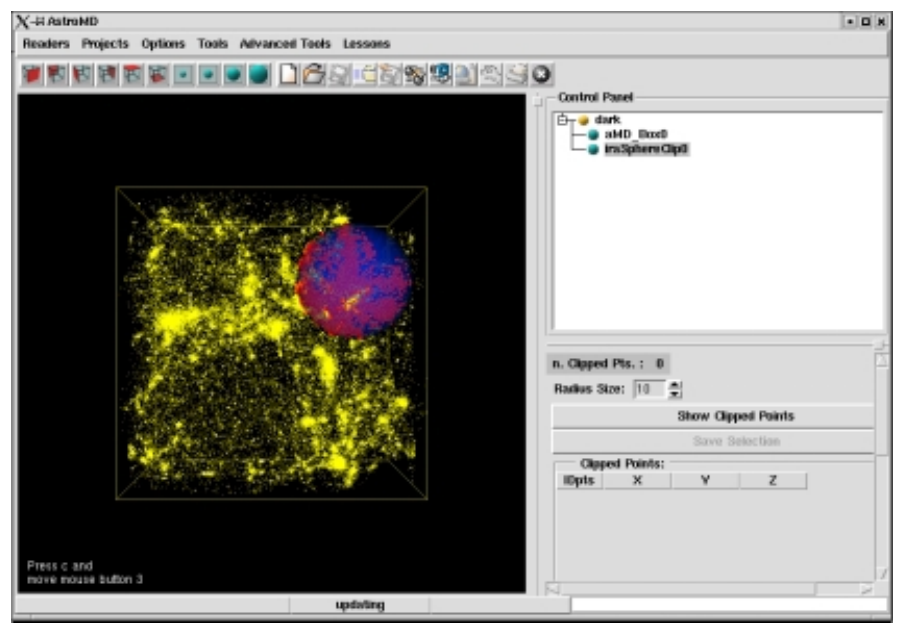

Fig. 1. The main GUI of AstroMD allows to visualize data (Render Window), to define variables and projects, to control variables and filters and to save results. The side of the cubic box is $50 \mathrm{Mpc}$ wide. The sample consists of 15000 particles extracted from a Nbody simulation of 16 millions of particles at redshift step $\mathrm{z}=0$, showing the formation of several structures.

analysis tools, like smoothing of the particle masses on a mesh or calculation of the power spectrum and correlation functions, are parts of the basic functionalities. AstroMD has also stereographic rendering capabilities, which makes it usable for immersive visualization, presently implemented at the Virtual Theatre of CINECA. This completes and improve its capabilities in the representation of three dimensional data set. Sitting in the chairs of the theatre and wearing 
stereoscopic glasses viewers can experience a semi-immersive Virtual Reality experience. Nevertheless this tool is exploited on different platforms, from the very sophisticated virtual theatre, down to the personal workstation. AstroMD, in fact, is developed with particular care to the portability issues in order to make it usable on many different platforms and to allow a large diffusion and usage inside the scientific community and educational institutions.

\subsection{Basic Functionalities}

AstroMD is thought to work with large datasets and uses advanced visualization tools, therefore it requires a corresponding powerful computational system. It suggested, at least, to use a PIII 500 PC with 256/512 Mbytes RAM memory, 100 Mbytes of free disk space and a high level graphic card. The required software consists in: Open GL (MSWindows) or Mesa (Linux) visualization libraries, TCL-TK 8.2 (or later), and the Visual ToolKit (VTK), which is distributed together with the AstroMD package. AstroMD allows to treat both particles (unstructured data) and continuous fields discretely represented over a computational mesh (structured data). Data must be written as sequences of $3 \mathrm{D}$ coordinates in the case of particles and as sequences of scalar values (fortran or $\mathrm{C}$ order) in the case of meshes. The input data Formats presently accepted by AstroMD is the common unformatted C standard, the Raw Format, the TIPSY and the FITS Formats. Raw files are simply dump of the memory, written in a continuous sequence of $\mathrm{x}, \mathrm{y}, \mathrm{z}$ coordinates with no labels or other symbols in within. TIPSY is a visualization toolkit specifically designed to quickly display and analyse the results of N-body simulations (http://www-hpcc.astro.washington.edu/tools/tipsy/tipsy.html). Tipsy requires a specific data Format to work with, supported also by AstroMD. The basic data structure is an array of particle structures in three separate arrays for each of the types of particle used in the simulations: collisionless particles, SPH particles, star particles and their characteristic properties, as potential energy and temperature. Binary and ASCII files can be read. Data are visualized with respect to a cubic box which describes the computational region. A cubic or spherical sub-region can be selected interactively inside the parent box with a different spatial resolution, in order to focus on the most interesting regions. Data inside the sampler can be studied with the analysed tools or can be saved in specific files for a off-line analysis. Boxes can be translated, rotated, zoomed in and out with respect to selected positions. Colours and luminosities can be chosen by the user. Images of different evolutionary stages can be combined in order to obtain a dynamic view of the behaviour of the system. The opacity of the particles can be increased, so that low density regions are more easily detectable, or decreased, so that the details of the high density regions substructures are shown. Different particles species (e.g. dark matter and baryons) can be visualizes at the same time using different colours. Other particles related continuous quantities, like density fields, can be calculated as typical grid bades fields and visualized as isosurfaces or volumes (Fig. 2). Scalars fields can be visualized by isosurfaces or by volume rendering. The value of the isosurface can be selected on 


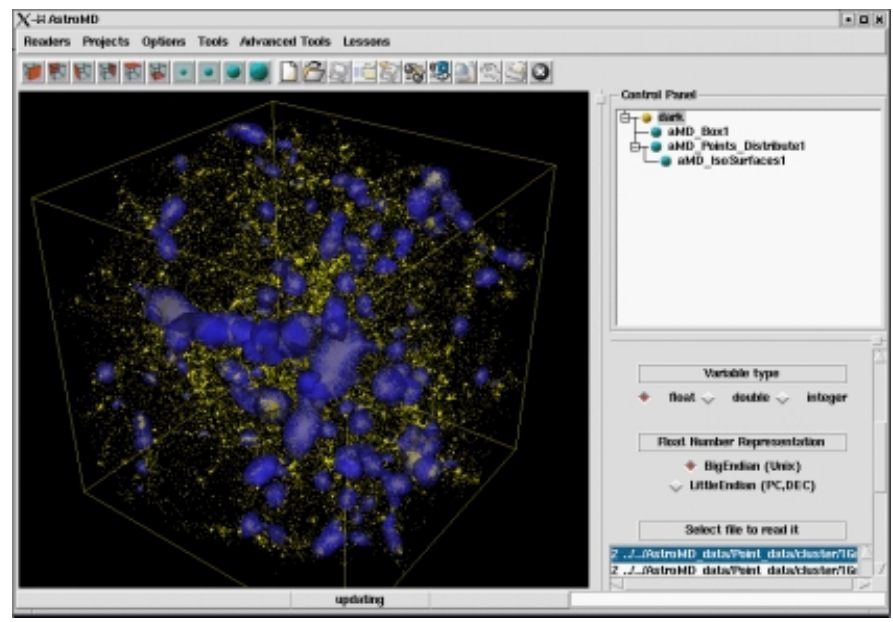

Fig. 2. Formation of clusters of galaxies in the universe, with overdensity of 200 with respect to the background density, during the evolution of a sample of 15000 particles in a cubic box of $50 \mathrm{Mpc}$.

the user interface. The volume rendering can be calculated using both the texture mapping and the ray tracing technique, depending on the specific hardware that is used (Fig. 3). Different time frames can be shown in a sequence. When particle-representation is used, the position of particles are interpolated between one time-step and the following. This improves the quality of the "animation" giving a fluidity otherwise unachievable. Both the single images and the whole sequence can be saved in bitmap or jpeg format to prepare an animation of the evolution. Enabling the steady-cam, the system can be rotated in Azimuth and Elevation during its evolution. Zoom-in and zoom-out possibilities are also offered. The whole set of particles can be visualized but it's also possible to use a sub-sample of the data, in order to get a faster and easier visualization. It was implemented a procedure which select randomly the sub-sample of data. Specific care has been devoted to avoid systematic errors in the selection procedure, so that the sample is still statistically significant.

\subsection{Data Analysis Functionalities}

Some built-in-tools, specifically directed to cosmological results of simulation, were implemented to allow an efficient manipulation and analysis of the data. At present the following functionality are implemented.

Particles mass density The mass density field associated to the particle distribution is calculated distributing the mass of each particle over the computational mesh by a eight points Cloud in Cell smoothing algorithm [?]. The computation can be done with the maximum accuracy using all the particles over a uniform 


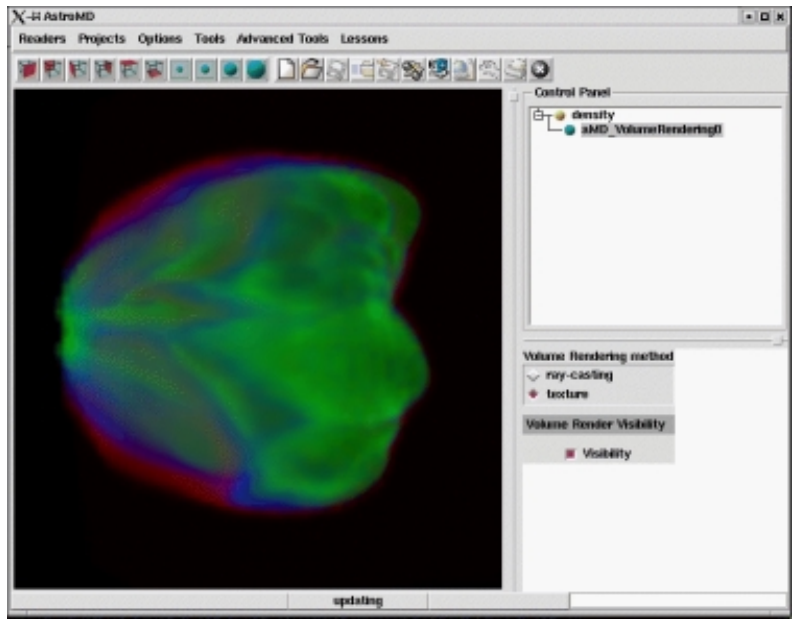

Fig. 3. Formation of clusters of galaxies in the universe, with overdensity of 200 with respect to the background density, during the evolution of a sample of 15000 particles in a cubic box of $50 \mathrm{Mpc}$.

high resolution mesh, but AstroMD allows the user to use only a sample of the whole set of particles, and the final result can be extrapolated to all particles of the simulation, reducing the CPU time consuming and the memory request. The smoothing of the masses can be performed generally using a coarse grid, that can be refined where high resolution is necessary.

The same tool can be used to calculate other fields related to quantities possibly associated to the particles, like, for example, the thermal energy density field or the X-ray luminosity field.

Gravitational field calculation Considering the mass density $\rho(x)$ defined over the computational mesh as above, the gravitational field can be calculated solving the Poisson equation

$$
\phi(\boldsymbol{x}) \propto \nabla^{2} \rho(\boldsymbol{x}),
$$

where $\phi(\boldsymbol{x})$ is the gravitational potential, by a Fourier Transform procedure. The Poisson equation is transformed in its momentum space image using a FFT VTK built-in function. This reduces the equation to a much simpler algrebric operation

$$
\phi(\boldsymbol{k}) \propto \frac{1}{\left|\boldsymbol{k}^{2}\right|} \rho(\boldsymbol{k}),
$$

where $\phi(\boldsymbol{k})$ and $\rho(\boldsymbol{k})$ are the Fourier images of the potential and of the density and $|\boldsymbol{k}|^{2}$ is the square module of the wavenumber. Finally, the potential is transformed to the physical space using an inverse FFT. 
Fourier decomposition, Power Spectrum and Correlation Function The quantity $\rho(\boldsymbol{k})$ is used to calculate the Power Spectrum $P(k)$ of the matter distribution, which is defined as the average value of the square norm of $\rho(\boldsymbol{k})$ :

$$
P(k)=\left\langle|\rho(\boldsymbol{k})|^{2}\right\rangle .
$$

The Power Spectrum expresses the weight of each of the Fourier components of the mass distribution between $k_{\min }$ and $k_{\max }$ which represents the inverse of the size of the computational mesh and the Nyquist frequency [3]. The Power Spectrum is a powerful measure of the statistical properties of the distribution, together with the associated Correlation Function $\xi(r)$, which is its Fourier Transform. The two-point Correlation Function indicates the probability to find

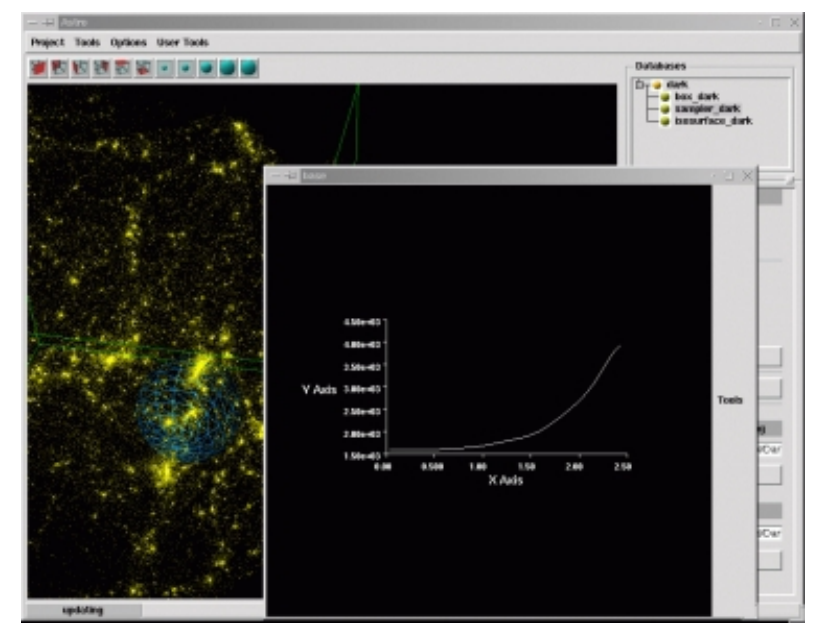

Fig. 4. Power Spectrum of the $\Lambda C D M$ simulation sample.

a particle at a distance $r$ from any other particle, and is usually used to analyze the clustering properties of a sample of discrete objects (particle, galaxies, galaxy clusters, etc) [1]. It was used HAM as his function estimator, because it is preferable at large scale, for samples with non uniform density [2]. The clustered zone is inserted in the sampler in which it is calculated the Correlation Function and the Power Spectrum. The plot $2 \mathrm{D}$ are shown in another window and is updated when the user points out to another clustered zone with mouse.

Minkowski Functionals The Minkowski Functionals provide a novel tool to characterize the large-scale distribution in the Universe [?]. They describe the Geometry, the Curvature and the Topology of a point set. Considering the set of points in 3D space, supplied by galaxies of a cluster of galaxies, and decorating each point with a ball of radius $r$, the tool measures the size, shape and 


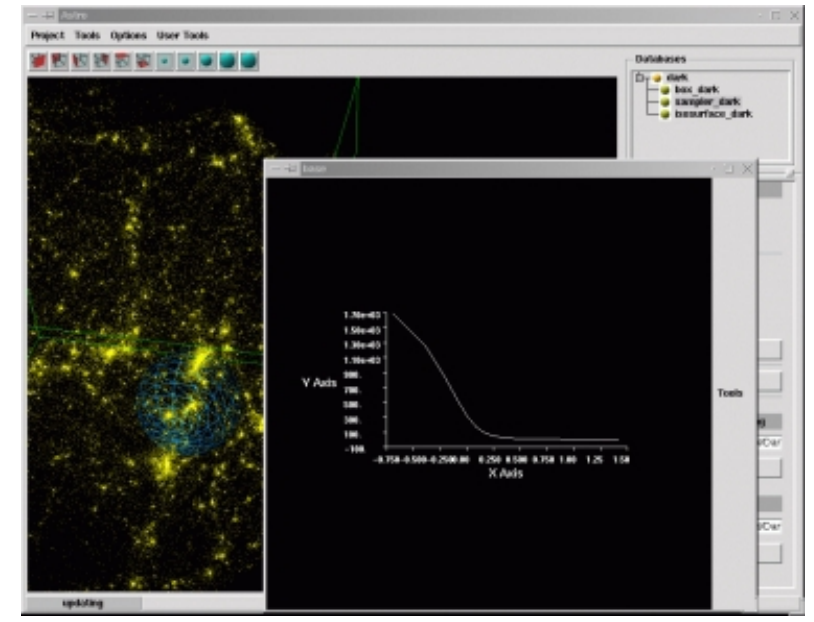

Fig. 5. Correlation Function of the $\Lambda C D M$ simulation sample.

connectivity of the spatial pattern formed by the union set of these balls. These characteristics change with the radius $r$, which may be employed as a diagnostic parameter.

Friend of Friend Algorithm It was inserted a group finder, known as Friendsof-Friends [?] (http//hpcc.astro.washington.edu/tools/). A particle belongs to a FoF group if it is within some linking length of any other particle in the group. After all such groups are found, those with less than a specified minimum number of group members are rejected. User must set two parameters: the maximum distance among particles forming clustering and numMembers, the minimum number of clustered particles. At last, FoF cancels all groups whose members are less than numMembers. It was also implemented the calculation of the centre of mass of each group, the number of components of groups and the radius of each group. The graphical output contains the grouped particles. The centre of mass of each group identified by ball with radius as clustered group radius. In another window is displayed the plot of the fraction of grouped particles versus the number of components of the groups. When the parameters are changed by user, FoF updates all its outputs.

\section{Conclusions}

AstroMD represents the first experience of a tool of immersive visualisation and data analysis in astrophysics. It will be a valuable tool for the scientific groups, which will be able to interact efficiently with large amount of data, easily 'navigating' inside them, analysing their properties, calculating their statistical properties and reconstructing their three dimensional shapes and features. This will 
1040 D. Ferro et al.

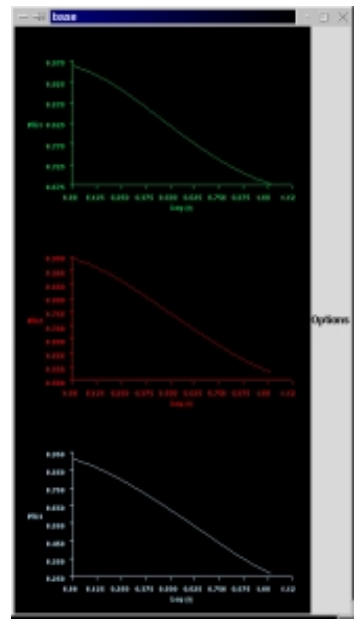

Fig. 6. Minkowski Functionals of a $\Lambda C D M$ simulation.

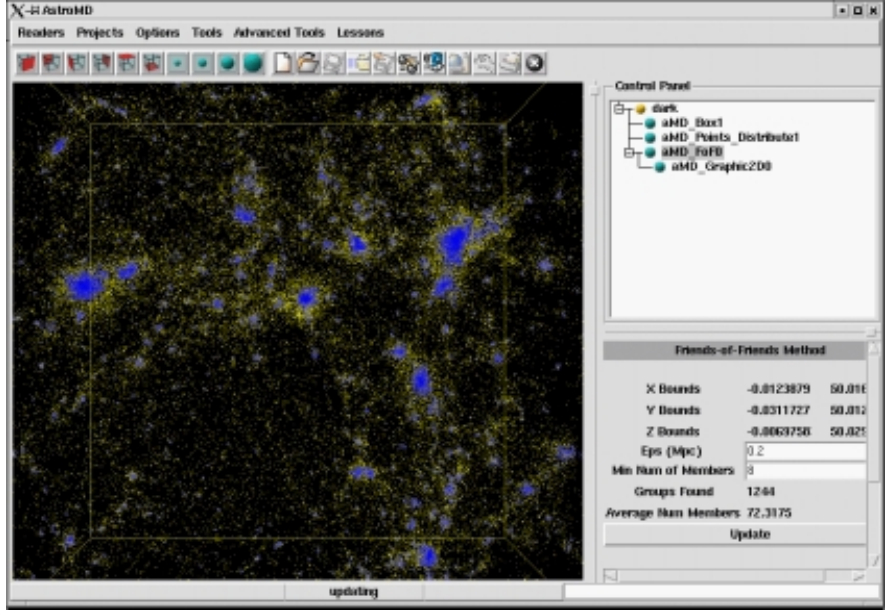

Fig. 7. FoF tool. 


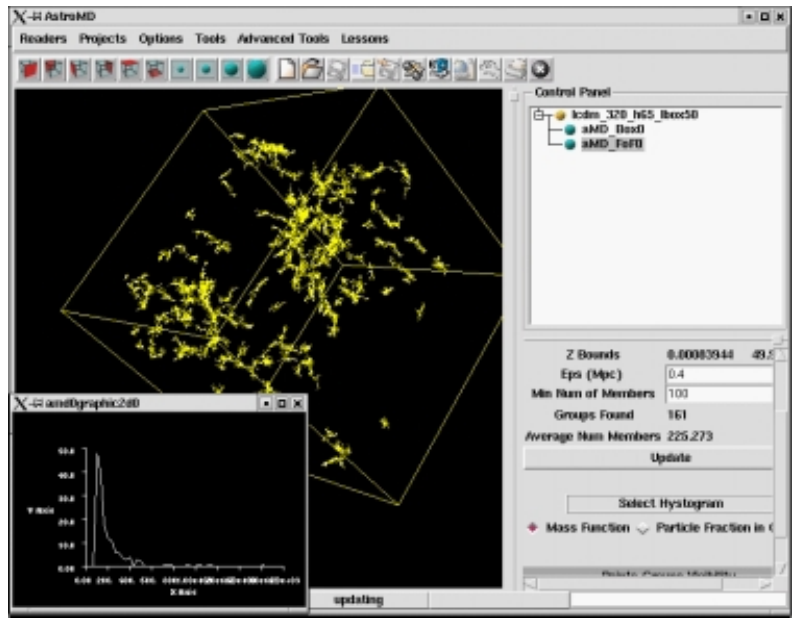

Fig. 8. FoF tool.

lead to a deeper understanding of the scientific problems and to their easier solution. The software developers and visualisation experts will have the possibility of strongly improving their knowledge in the involved techniques and their experience on the possible applications of 3D visualisation and immersive technology in the scientific research applications. Finally, the use of AstroMD for educational purposes will be a very effective way of presenting astrophysical problems to students, giving the possibility of observing objects and structures from a privileged and personalised.

\section{References}

1. Blanchard, A., Alimi, J.: Practical determination of the spatial correlation function. Astron. Astrophys. 203 (1988) L1-L4

2. Pons-Bordería, M.J., Martínez, V.J. et al: Comparing estimators of the galaxy correlation function. ApJ 523 (1999) 480-491

3. Jenkins, A., Frenk, C.S., Pearce, F.R. et al: Evolution of Structure in Cold Dark Matter Universes. Astrophys. J. 499 (1998) 20

4. Schroeder, W., Ken M., Lorensen B.: The Visualization Toolkit. Prentice Hall (1999)

5. Rabinowitz, P.: On subharmonic solutions of a Hamiltonian system. Comm. Pure Appl. Math. 33 (1980) 609-633 